\title{
Special Issue on Reintegrating Artificial Intelligence and Robotics
}

\author{
Federico Pecora ${ }^{1} \cdot$ Masoumeh Mansouri ${ }^{2} \cdot$ Nick Hawes $^{3} \cdot$ Lars Kunze $^{3}$ \\ ๑) Gesellschaft für Informatik e.V. and Springer-Verlag GmbH Germany, part of Springer Nature 2019
}

Making robots intelligent means rendering them capable of reasoning about their environment, planning to achieve goals, enacting plans in the real world, and reacting to unforeseen events. The field of artificial intelligence (AI) was born of the need to provide these skills, and has since branched into different sub-disciplines, including perception, knowledge representation and reasoning, planning, interaction, and learning. Research in these fields has led to fantastic innovations, many of which have been featured in special issues of the KI journal. Yet, despite more than half a century of innovations, AI has not fully achieved the promise of making robots intelligent. Researchers in AI have clustered around abstractions of real-world problems. Often, these reflect simplifying assumptions that do not hold in the case of physically situated and embodied robot systems. Difficult and diverse challenges emerge from the need to use AI methods with and for robot systems, including bridging the percept-symbol gap, interacting with and learning from humans, integrating task and motion planning, and combining systems to build complete robotic solutions.

Several of the above challenges are represented in the technical papers collected in this special issue. Neubert et al. investigate hyper-dimensional computing to perform symbolic operations in different robot tasks including object

Lars Kunze

lars@ robots.ox.ac.uk

Federico Pecora

federico.pecora@oru.se

Masoumeh Mansouri

m.mansouri@bham.ac.uk

Nick Hawes

nickh@ robots.ox.ac.uk

1 Center for Applied Autonomous Sensor Systems, Örebro University, Örebro, Sweden

2 School of Computer Science, University of Birmingham, Birmingham, UK

3 Oxford Robotics Institute, Department of Engineering Science, University of Oxford, Oxford, UK recognition, place recognition, and learning of simple reactive behaviours. Sridarahn and Meadows cover topics related to how robots explain themselves when collaborating with humans. In their paper, they present a theory of explanations, an architecture that implements the theory on robots, as well as simulated robot experiments. The complex process of building a complete robot system that interacts with humans in a domestic environment is described by Lima et al., while Basiri et al. contribute their experience in systematically evaluating and benchmarking service robots through organising recurrent competitions under the European Robotics League.

In his recent $\mathrm{PhD}$ thesis, Wulfmeier focused on datadriven machine learning approaches in relation to how robots learn to perceive and act in their environment. In particular, he studied the problem of supervision for robot learning via imitation, simulation and adaptation. His dissertation abstract is included in this special issue.

The combination of AI methods and physical robot systems is paramount to industrial applications. This special issue includes three testimonials from industry related to AI transfer. Davies describes where we are and what is missing to achieve effective warehouse automation solutions, while Amend et al. illustrate the challenges and opportunities for AI in weed management. The perspective of Volvo Trucks is provided by Götvall, who describes the challenges facing industrial production and how academia and industry can address them in cooperation.

In her discussion, Kirsch provides an academic perspective on the problem of combining AI methods and physical robot systems. She questions the assumptions commonly made in robotics and AI, and discusses how to foster step changes in these research communities.

Finally, this special issue features an interview with Aaron Sloman, whose work has provided key insights for reintegrating AI and robotics. He reflects here on the design principles of robots and the need for adequate learning mechanisms, and how these are related to embodiment. Further, he talks about the testability of robot intelligence, real-world applications, and future challenges in the field. 
The contributions collected in this special issue can be read from three different perspectives: that of an AI researcher interested in developing methods for use in real robot systems; that of a roboticist who wants to know what AI can do for his or her robot; last, but certainly not least, that of a practitioner who is interested in intelligent robotic solutions for solving real-world problems. Whichever category describes you best, we thank you for reading, and we hope that this special issue of the KI journal will stimulate your future work!

\section{Content}

This special issue is composed of technical contributions, doctoral dissertation abstracts, AI Transfer articles, a discussion, and an interview. Published contributions are from research labs and companies located in Portugal, Sweden, United Kingdom, and Germany.

\subsection{Technical Contributions}

- An Introduction to Hyperdimensional Computing for Robotics

Peer Neubert, Stefan Schubert and Peter Protzel

- Towards a Theory of Explanations for Human-Robot Collaboration

Mohan Sridharan and Ben Meadows

- SocRob@Home Integrating AI Components in a Domestic Robot System

Pedro U. Lima, Carlos Azevedo, Emilia Brzozowska, João Cartucho, Tiago Dias, João Gonçalves, Mithun Kinarullathil, Guilherme Lawless, Oscar Lima, Rute Luz, Pedro Miraldo, Enrico Piazza, Miguel Silva, Tiago Veiga, and Rodrigo Ventura

- Benchmarking Functionalities of Domestic Service Robots Through Scientific Competitions

Meysam Basiri, Enrico Piazza, Matteo Matteucci, and Pedro U. Lima

\subsection{Dissertation Abstracts}

- Efficient Supervision for Robot Learning via Imitation, Simulation, and Adaptation

Markus Wulfmeier

\subsection{Al Transfer}

- Deskilling Robots in Logistics Environments Martin Raymond Davies

- Weed Management of the Future

Sandra Amend, David Brandt, Daniel Di Marco, Tobias Dipper, Gabriel Gässler, Maurice Gohlke,
Markus Höferlin, Katharina Kesenheimer, Peter Lindner, Roland Leidenfrost, Anette Mezger, Andreas Michaels, Tobias Mugele, Arthur Müller, Tanja Riffel, Yeshwanth Sampangi, and Jan Winkler

- Volvo Group Collaborative Robot Systems laboratory A collaborative way for the academia and industry to be at the forefront of Artificial Intelligence

Per-Lage Götvall

\subsection{Discussions}

- Shakey ever after? Questioning tacit assumptions in robotics and artificial intelligence

Alexandra Kirsch

\subsection{Interviews}

- A Philosophically Motivated View on AI and Robotics-Interview with Aaron Sloman, Honorary Professor of Artificial Intelligence and Cognitive Science, University of Birmingham, United Kingdom

Lars Kunze and Aaron Sloman

\section{Service}

\subsection{Conferences and Workshops}

- IEEE International Conference on Robotics and Automation (ICRA), https://www.icra2020.org

- IEEE/RSJ International Conference on Intelligent Robots and Systems (IROS), http://www.iros2020.org

- Robotics: Science and Systems (RSS), http://www. roboticsconference.org

- International Joint Conference on Artificial Intelligence (IJCAI), https://ijcai20.org

- European Conference on Artificial Intelligence (ECAI), http://ecai2020.eu

- AAAI Conference on Artificial Intelligence (AAAI), https://aaai.org/Conferences/AAAI-20/

- International Joint Conference on Autonomous Agents and Multi-Agent Systems (AAMAS), https://aamas 2020.conference.auckland.ac.nz

\subsection{Journals}

- IEEE Robotics Automation Magazine (RAM), https:// www.ieee-ras.org/publications/ram

- IEEE Robotics and Automation Letters (RA-L), https ://www.ieee-ras.org/publications/ra-1 
- Robotics and Autonomous Systems (RAS), https:// www.journals.elsevier.com/robotics-and-autonomous -systems

- Intelligent Service Robotics, https://www.springer.com/ engineering/control/journal/11370

- Artificial Intelligence, http://www.journals.elsevier.com/ artificial-intelligence
- Journal of Artificial Intelligence Research, http://jair.org

- AI Perspectives, https://aiperspectives.springeropen.com 